\title{
Market-Based Reinforcement Learning in Partially Observable Worlds
}

\author{
Ivo Kwee, Marcus Hutter and Jürgen Schmidhuber \\ IDSIA, Manno CH-6928, Switzerland. \\ \{ivo, marcus, juergen\}@idsia.ch *
}

\begin{abstract}
Unlike traditional reinforcement learning (RL), market-based RL is in principle applicable to worlds described by partially observable Markov Decision Processes (POMDPs), where an agent needs to learn short-term memories of relevant previous events in order to execute optimal actions. Most previous work, however, has focused on reactive settings (MDPs) instead of POMDPs. Here we reimplement a recent approach to market-based RL and for the first time evaluate it in a toy POMDP setting.
\end{abstract}

\section{Introduction}

One major reason for the importance of methods that learn sequential, event-memorizing algorithms is this: sensory information is usually insufficient to infer the environment's state (perceptual aliasing, Whitehead 1992). This complicates goal-directed behavior. For instance, suppose your instructions for the way to the station are: "Follow this road to the traffic light, turn left, follow that road to the next traffic light, turn right, there you are." Suppose you reach one of the traffic lights. To do the right thing you need to know whether it is the first or the second. This requires at least one bit of memory - your current environmental input by itself is not sufficient. Any learning algorithm supposed to learn algorithms that store relevant bits of information from training examples that only tell whether the current attempt has been successful or not - this is the typical reinforcement learning (RL) situation - will face a major temporal credit assignment problem: which of the many past inputs are relevant (and should be represented in some sort of internal memory), which are not? Most traditional work on RL requires Markovian interfaces to the environment: the current input must provide all information about probabilities of possible inputs to be observed after the next action, e.g., [15]16]. These approaches, however, essentially learn how to react in response to a given input, but cannot learn to identify and memorize important past events in complex, partially observable settings, such as in the introductory example above.

Several recent, non-standard RL approaches in principle are able to deal with partially observable environments and can learn to memorize certain types of relevant events [7,8,6, 3, 9, 10, 13, 18, 17, 14. None of them, however, represents a satisfactory solution to the general problem of learning in worlds described by partially observable Markov Decision Processes

\footnotetext{
* This work was supported by SNF grants 21-55409.98 and 2000-61847.00
} 
(POMDPs). The approaches are either ad hoc, or work in restricted domains only, suffer from problems concerning state space exploration versus exploitation, have non-optimal learning rate or have limited generalization capabilities. Most of these problems are overcome in [5] but the model is yet computationally intractable. Here we consider a novel approach to POMDPRL called market-based $R L$ which in principle does not suffer from the limitations of traditional RL. We first give an overview of this approach and its history, then evaluate it in a POMDP setting, and discuss its potential and limitations.

\section{Market-based RL: History \& State of the Art}

Classifier systems. Probably the first market-based approach to RL is embodied by Holland's Classifier Systems and the Bucket Brigade algorithm

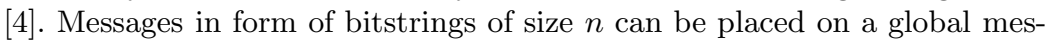
sage list either by the environment or by entities called classifiers. Each classifier consists of a condition part and an action part defining a message it might send to the message list. Both parts are strings out of $\{0,1,\}^{n}$ where the '.' serves as a 'don't care' if it appears in the condition part. A non-negative real number is associated with each classifier indicating its 'strength'. During one cycle all messages on the message list are compared with the condition parts of all classifiers of the system. Each matching classifier computes a 'bid' based on its strength. The highest bidding classifiers may place their message on the message list of the next cycle, but they have to pay with their bid which is distributed among the classifiers active during the last time step which set up the triggering conditions (this explains the name bucket brigade). Certain messages result in an action within the environment (like moving a robot one step). Because some of these actions may be regarded as 'useful' by an external critic who can give payoff by increasing the strengths of the currently active classifiers, learning may take place. The central idea is that classifiers which are not active when the environment gives payoff but which had an important role for setting the stage for directly rewarded classifiers can earn credit by participating in 'bucket brigade chains'. The success of some active classifier recursively depends on the success of classifiers that are active at the following time ticks. Bankrupt classifiers are removed and replaced by freshly generated ones endowed with an initial amount of money.

PSALMs. Holland's approach suffers from certain drawbacks though. For instance, there is no credit conservation law - money can be generated out of nothing. Pages 23-51 of 11] are devoted to a more general approach called Prototypical Self-referential Associating Learning Mechanisms (PSALMs). Competing/cooperating agents bid for executing actions. Winners may receive external reward for achieving goals. Agents are supposed to learn the credit assignment process itself (meta-learning). For this purpose they can execute actions for collectively constructing and connecting and modifying agents and for transferring credit (reward) to agents. PSALMs are the first systems that enforce the important constraint of total credit conservation 
(except for consumption and external reward) - this constraint is not enforced in Holland's classifier economy, which may cause money inflation and other problems. PSALMs also inspired the money-conserving Neural bucket brigade, where the money is "weight substance" of a reinforcement learning neural net [12].

Hayek machines [⿰] [- are designed to avoid further loopholes in Holland's credit assignment process that may allow some agent to profit from actions that are not beneficial for the system as a whole. Property rights of agents are strictly enforced. The current owner of the right to act in the world computes the minimal price for this right, and sells it to the highest bidder. Agents can create children and invest part of their money into them, and profit from their children's success. Wealth and bid of an agent are separate quantities. All of this makes Hayek more stable than the original bucket brigade. There have been impressive applications - Hayek learned to solve complex blocks world problems [1]. Hayek is reminiscent of PSALM3 11, but a crucial difference between PSALM3 and Hayek may be that PSALM3 does not strictly enforce individual property rights. For instance, agents may steal money from other agents and temporally use it in a way that does not contribute to the system's overall progress.

\section{The Hayek4 System}

We briefly describe our reimplementation of Hayek4 [2] - the most recent Hayek machine variant. Unlike earlier Hayek versions, Hayek4 uses a new representation language.

Economic model. Hayek4 consists of a collection of agents that each have a rule, a possible action, a wealth and a numerical bid. The agents are required to solve an instance of a complex task. But because each agent can only perform a single action, they need to cooperate. In a single instance, computation proceeds in a series of auctions. The highest bidding agent wins and "owns" the world, pays an amount equal to its bid to the previous owner and may then perform its action on the world. The owner of the world collects any reward from it, plus the payment of the next owner. Only if the actual revenue is larger than its bid, an agent can increase its wealth.

Birth, death and taxes. Agents are allowed to create children if their wealth is larger than some predetermined number (here 1000). Parents endorse their offspring with a minimum amount of money which is subtracted from their wealth, but in return parents receive a share $c$ of their children's future profit. Children's rules are randomly created, copied or mutated from their parents with probabilities $p_{r}, p_{c}$ and $p_{m}$, respectively. See Table 1 for their numerical values.

By the definition of wealth, birth processes automatically focus on rather successful agents. To improve efficiency, after each instance all agents pay a small amount of tax proportional to the amount of computation time they have used. To remove unuseful agents, earlier Hayek versions required agents to pay a small fixed amount of tax; agents without money were removed from the system. Hayek4 removes them once they have been inactive for the last, say, 100 instances. 


\begin{tabular}{lll}
\multicolumn{2}{l}{ symbol value description } \\
\hline$W$ & 10.0 & Baum and Durdanovic's $W$ parameter \\
$R$ & 100.0 & reward value \\
$\epsilon$ & 0.01 & children always bid this amount higher than highest bid \\
$u$ & 0.25 & increase level if $(1-u)$ instances are solved; decrease if less than $u$. \\
$t$ & $1 \mathrm{e}-3$ & execution tax \\
$c$ & 0.25 & copyright share \\
$m$ & 0.5 & mutation ratio \\
$p_{r}$ & 0.3 & probability of random rule for new child \\
$p_{c}$ & 0.3 & probability of copying rule for new child \\
$p_{m}$ & 0.3 & probability of mutation of rule for new child \\
: Parameter & Pettings for our Hayek4 reimplementation. For a detailed explanation see
\end{tabular}

Table 1: Parameter settings for our Hayek4 reimplementation. For a detailed explanation see 2 .

Post production system. The agents in Hayek4 are essentially a Post production system. Baum and Durdanovic argue that this provides a richer representation than the S-expressions in earlier Hayek versions because Post systems are Turing complete (like the representation used by PSALMs [11]). Each agent forms a Post rule of the form $L \rightarrow R$ where $L$ is the antecedent (or rule) and $R$ is the consequent (the action). The state is encoded in a string $S$; the agent is only allowed to perform $R$ when $L$ matches string $S$. This procedure is iterated until no rules match, and computation halts.

\section{Implementation}

We found Hayek4 rather difficult to reproduce. Several parameters require tuning — making Hayek stable still seems to remain an art. Parameter settings of our Hayek system are summarized in Table 1; for a detailed explanation see [2]. For small task sizes our program tends to find reliable solutions, but larger sizes cause stability problems, or prevent Hayek4 from finding a solution within several days.

We tried to reimplement Hayek4 as closely as possible, except for certain deviations justified by improved performance or implementation issues:

- Single rule: instead of agents having multiple rules, we allowed only one rule per agent.

- Fixed reward: instead of paying off reward proportional to the size of the task, we used a fixed reward of 100 .

- TD backup: we additionally applied time differential (TD) 15 backup of the agent's bid, as traditionally done in reinforcement learning systems, to accelerate convergence.

Blockworld. Baum and Durdanovic reported a set of Hayek agents that collectively form a universal solver for arbitrary blockworld problems 2 2 involving arbitrarily high stacks of blocks. The set consists of five rules listed in the table below.

We tested Hayek 4 by first hardwiring a system consisting of those five socalled universal agents only. Indeed, it reliably generated stacks of increasing size, reaching stack level 20 within 800 instances, and level 30 within 1000 instances. Figure 2 shows a typical run resulting in a stack of height $=4$. 


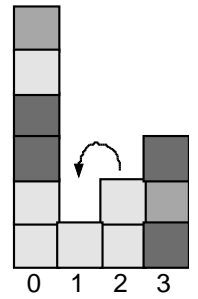

Fig. 1: Example of Blockworld instance with stack height $=6$. The objective is to replicate in stack 1 the color sequence represented by the blocks in stack 0 , by moving blocks between stacks 1-3. Here the optimal next move would be to move the top block of stack 2 to stack 1 .

\begin{tabular}{|c|c|c|c|}
\hline \multirow[b]{2}{*}{ world } & \multicolumn{3}{|c|}{ agent } \\
\hline & rule & actior & bid \\
\hline babc:cbb::a & $*: *: *: *$ & $1 \rightarrow 3$ & 7.78 \\
\hline babc:cb: :ab & $*: *: *: *$ & $1 \rightarrow 3$ & 7.78 \\
\hline babc:c: :abb & $*: *: *: *$ & $1 \rightarrow 3$ & 7.78 \\
\hline babc: : :abbc & $* . *: 1: *: * 2 *$ & $3 \rightarrow 2$ & 8.07 \\
\hline babc: : c:abb & $* . *: 1: *: * 2 *$ & $3 \rightarrow 2$ & 8.07 \\
\hline babc: : cb:ab & $* . *: 1: * 2: *$ & $2 \rightarrow 1$ & 8.07 \\
\hline$b a b c: b: c: a b$ & $* . *: 1: *: * 2 *$ & $3 \rightarrow 2$ & 8.07 \\
\hline$b a b c: b: c b: a$ & $* . *: 1: *: * 2 *$ & $3 \rightarrow 2$ & 8.07 \\
\hline babc:b:cba: & $* . *: 1: * 2: *$ & $2 \rightarrow 1$ & 8.07 \\
\hline babc:ba:cb: & $* . *: 1: * 2: *$ & $2 \rightarrow 1$ & 8.07 \\
\hline babc:bab:c: & $* .: 1: * 2: *$ & $2 \rightarrow 1$ & 35.80 \\
\hline babc: babc: : & & & \\
\hline
\end{tabular}

Fig. 2: A typical Blockworld solution for stack height $=4$. Each colon-separated field of the world string represents a stack of blocks of colors $\{a, b, c\}$. The agent's rule matches the world string; "*" represents a "don't care" symbol; numbers in the rule encode replacements of matched substrings of stack 0 . Actions move blocks between stacks 1,2 and 3 .

Then we switched on the creation of children but kept mutation turned off. This caused Hayek to progress at a much slower pace, probably because new children disrupt the universal solver as they always win the auctions using the $\epsilon$-bid scheme.

Problems. Unfortunately, our system was not able to find the universal solver by itself. It typically reached stack size 5 , but then failed to progress any further. We believe that with increasing task size the system is encountering stability problems. Much remains to be done here.

\section{$5 \quad$ Adding Memory to Hayek}

Unlike traditional RL, market-based RL is in principle applicable to POMDPs. Previous work, however, has usually focused on reactive settings (MDPs) instead of POMDPs (see 19] for a notable exception though). Here we add a memory register to the Hayek machine and apply it to POMDPs. The memory register acts as a additional environmental variable that can be manipulated by the agent using write actions.

Test problem. To study Hayek4's performance on POMDPs, we focus on one of the simplest possible POMDP problems. Figure 3 shows our toy example which is an adapted version of Woods101 [3], a standard test case for non-Markov search problems. Agents start in either of the two positions, $L$ or $R$, and have to reach the food at $F$. Agents have only limited perception of their four immediate neighbouring positions north, east, south and west plus the value of the memory register. Without memory, there is no Markov solution to this problem because there are two aliased positions $A$ and $B$ with identical environmental input where the optimal agent needs to execute different actions: in $A$ the optimal action is east; in $B$ the optimal action is west. Note that the starting positions $L$ and $R$ are also aliased. However, 


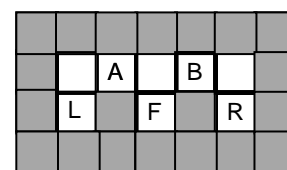

maze

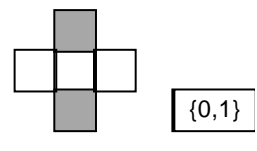

sensation

Fig. 3: Woods101 is a partially observable environment; grey positions represent walls. Left: The agent starts at one of the positions $L$ or $R$ and has to reach the food at $F$. The two aliased positions are $A$ and $B$. A 1-bit memory register is available for writing/reading. Right: the agent senses the four neigbouring positions and the value of the memory register; here, for example, the agent might be either at $A$ or at $B$.

\begin{tabular}{ccccccccc}
\multicolumn{3}{c}{ state } & & \multicolumn{4}{c}{ agent } \\
\cline { 1 - 5 } \cline { 6 - 8 } x & $\mathrm{y}$ & $\mathrm{m}$ & & rule & action & bid wealth \\
\hline 1 & 1 & 0 & & $1: 0: 0: 0: 0$ & $\mathrm{~m} 1$ & 100.00 & 119.51 \\
1 & 1 & 1 & & $1:^{*}: *: 0: 1$ & $\mathrm{n}$ & 100.00 & 184.04 \\
1 & 2 & 1 & & $*^{*}: 1: 0: 1$ & $\mathrm{~m} 0$ & 100.00 & 301.70 \\
1 & 2 & 0 & & $0: 1: 1: 0: 0$ & $\mathrm{e}$ & 100.00 & 116.76 \\
2 & 2 & 0 & & $0: 1: 0: 1: 0$ & $\mathrm{e}$ & 100.00 & 951.08 \\
3 & 2 & 0 & & $0: 1: 1: 1:^{*}$ & $\mathrm{~s}$ & 100.00 & 103.52 \\
3 & 1 & 0 & & & & &
\end{tabular}

\begin{tabular}{|c|c|c|c|c|c|}
\hline \multicolumn{2}{|c|}{ state } & \multicolumn{4}{|c|}{ agent } \\
\hline $\mathrm{xy}$ & $\bar{m}$ & rule & action & bid & wealth \\
\hline 11 & 1 & $1:^{*}: *: 0: 1$ & $\mathrm{n}$ & 100.00 & 185.01 \\
\hline 12 & 1 & $*: *: 1: 0: 1$ & $\mathrm{~m} 0$ & 100.00 & 302.15 \\
\hline 12 & 0 & $0: 1: 1: 0: 0$ & $\mathrm{e}$ & 100.00 & 117.22 \\
\hline 22 & 0 & $0: 1: 0: 1: 0$ & $\mathrm{e}$ & 100.00 & 951.40 \\
\hline 32 & 0 & $0: 1: 1: 1:^{*}$ & $\mathrm{~s}$ & 100.00 & 103.51 \\
\hline
\end{tabular}

Table 2: Two policies from starting position $L=(1,1)$; auctions proceed from the first line downwards. Left: the memory is set to $m=1$ before going north but again reset to $m=0$ before the aliased position $(2,2)$ is reached. Right: the memory is initially set to $m=0$ by a memory agent at $(1,2)$. The first four fields of the rule represent the bit values of north, east, south and west, respectively; the fifth field matches the value of the memory register. Star ('*') variables represent "don't care" symbols.

in both $L$ and $R$ the optimal action is coincidentally the same (i.e. going north).

Results. We ran Hayek4 on our test problem. After about 1000 instances it learned a policy that solved the POMDP problem using a 1-bit memory. In early instances the number of agents grew up to 1000 or more but finally only 8 agents remained. While the bids of all agents converged to the actual reward value of 100 , their wealth values varied considerably. Sample histories of solved instances starting at $L$ are shown in Table 2 ; histories of solved instances starting at $R$ are shown in Table 3 .

The results can be summarized as follows. When an instance starts from $L$, Hayek assures that the memory is set to 0 before it reaches aliased position $A$. If the memory was initially set to $m=1$, a write agent sets the memory to 0 ; if the memory was already $m=0$, nothing is done. When the agent starts from the right, the opposite occurs: before Hayek reaches aliased position $B$, it checks if the memory bit is properly set to $m=1$. Now, two different agents - with identical perception of the environment but different perception of the memory - act at aliased positions $A$ and $B$ : an east agent at $A$ if the memory bit is 0 , and a west agent if the memory is set to $m=1$.

Hayek also evolves one agent executing action south at sequence ends at $(3,2)$. It disregards the value of the memory by using the '*'-symbol ("don't care"). "Don't care" symbols are useful to prevent unnecessary over-specialization, i.e., we do not need two separate agents for each memory value. In fact, such over-specialization occurred with the starting agent going north: 


\begin{tabular}{|c|c|c|c|c|c|}
\hline \multicolumn{2}{|c|}{ state } & \multicolumn{4}{|c|}{ agent } \\
\hline$x y$ & $\mathrm{~m}$ & rule & action & bid & wealth \\
\hline 51 & 1 & $1:^{*}:^{*}: 0: 1$ & $\mathrm{n}$ & 100.00 & 183.80 \\
\hline 52 & 1 & $0: 0: 1: 1: 1$ & $\mathrm{w}$ & 100.00 & 143.04 \\
\hline 42 & 1 & $0: 1: 0:^{*}: 1$ & $\mathrm{w}$ & 100.00 & 378.54 \\
\hline 32 & 1 & $0: 1: 1: 1:^{*}$ & $\mathrm{~s}$ & 100.00 & 103.52 \\
\hline 31 & 1 & & & & \\
\hline
\end{tabular}

\begin{tabular}{|c|c|c|c|c|}
\hline \multicolumn{2}{|c|}{ state } & \multicolumn{3}{|c|}{ agent } \\
\hline $\mathrm{xy}$ & $\mathrm{m}$ & rule & action & bid wealth \\
\hline 51 & 0 & $1: 0: 0: 0: 0$ & $\mathrm{~m} 1$ & 100.00120 .21 \\
\hline 52 & 1 & $1:^{*}: *: 0: 1$ & $\mathrm{n}$ & 100.00185 .33 \\
\hline 52 & 1 & $0: 0: 1: 1: 1$ & $\mathrm{w}$ & $100.00 \quad 143.75$ \\
\hline 42 & 1 & $0: 1: 0:^{*}: 1$ & $\mathrm{w}$ & 100.00379 .05 \\
\hline 32 & 1 & $0: 1: 1: 1:^{*}$ & $\mathrm{~s}$ & $100.00 \quad 103.50$ \\
\hline 31 & 1 & & & \\
\hline
\end{tabular}

Table 3: Two policies from starting position $R=(5,1)$; auctions proceed from the first line downwards. Left: the memory is initially set to $m=1$ and the agent proceeds directly north to $F=(5,2)$. Right: the memory was initially set to $m=0$ and a memory agent sets $m=1$ before proceeding north.

Hayek did not use a "don't care" symbol for the memory register and therefore needed a extra memory agent $(m 1)$ if the bit was initially unset.

Discussion. This simple test problem shows that Hayek, in principle, is able to solve POMDPs. More precisely, using a memory register, Hayek "de-aliases" aliased positions in the POMDP by tagging these situations before they actually occur. We note that the solution found by Hayek is not unique: it does not matter whether it chooses to write a "0" or a "1" for the left path, as long as the right path uses the complement. Once a policy seems to perform well, Hayek converges toward this local optimum and sticks to it.

Note that the found solution is nearly but not exactly optimal; an extra $m 1$ memory agent was necessary for the left policy in Table 2 because Hayek was not able to generalize optimally in case of the agent going north, failing to use a "don't care" symbol for the memory value.

\section{Conclusion}

We have started to evaluate market-based RL in POMDP settings, focusing on the Hayek machine as a vehicle for learning to memorize relevant events in short-term memory. Using a memory bit register, Hayek was able to distinguish aliased positions in a toy POMDP and evolve a stable solution. The approach is promising, yet much remains to be done to make it scalable.

\section{References}

1. E. B. Baum and I. Durdanovic. Toward a model of mind as an economy of agents. Machine Learning, 35(2):155-185, 1999.

2. E. B. Baum and I. Durdanovic. An evolutionary Post production system. Technical report, NEC Research Institute, Princeton, NJ, January 2000.

3. D. Cliff and S. Ross. Adding temporary memory to ZCS. Adaptive Behavior, 3:101-150, 1994.

4. J. H. Holland. Properties of the bucket brigade. In Proceedings of an International Conference on Genetic Algorithms. Hillsdale, NJ, 1985. 
5. M. Hutter. Towards a universal theory of artificial intelligence based on algorithmic probability and sequential decision theory. Technical Report IDSIA-14-00, IDSIA, Manno, Switzerland, December 2000. ftp.idsia.ch/pub/techrep/IDSIA-14-00.ps.gz.

6. T. Jaakkola, S. P. Singh, and M. I. Jordan. Reinforcement learning algorithm for partially observable Markov decision problems. In G. Tesauro, D. S. Touretzky, and T. K. Leen, editors, Advances in Neural Information Processing Systems 7, pages 345-352. MIT Press, Cambridge MA, 1995.

7. L.P. Kaelbling, M.L. Littman, and A.R. Cassandra. Planning and acting in partially observable stochastic domains. Technical report, Brown University, Providence RI, 1995.

8. M.L. Littman, A.R. Cassandra, and L.P. Kaelbling. Learning policies for partially observable environments: Scaling up. In A. Prieditis and S. Russell, editors, Machine Learning: Proceedings of the Twelfth International Conference, pages 362-370. Morgan Kaufmann Publishers, San Francisco, CA, 1995.

9. R. A. McCallum. Overcoming incomplete perception with utile distinction memory. In Machine Learning: Proceedings of the Tenth International Conference. Morgan Kaufmann, Amherst, MA, 1993.

10. M. B. Ring. Continual Learning in Reinforcement Environments. PhD thesis, University of Texas at Austin, Austin, Texas 78712, August 1994.

11. J. Schmidhuber. Evolutionary principles in self-referential learning, or on learning how to learn: the meta-meta-... hook. Institut für Informatik, Technische Universität München, 1987.

12. J. Schmidhuber. A local learning algorithm for dynamic feedforward and recurrent networks. Connection Science, 1(4):403-412, 1989.

13. J. Schmidhuber. Reinforcement learning in Markovian and nonMarkovian environments. In D. S. Lippman, J. E. Moody, and D. S. Touretzky, editors, Advances in Neural Information Processing Systems 3, pages 500-506. San Mateo, CA: Morgan Kaufmann, 1991.

14. J. Schmidhuber, J. Zhao, and M. Wiering. Shifting inductive bias with success-story algorithm, adaptive Levin search, and incremental selfimprovement. Machine Learning, 28:105-130, 1997.

15. R. S. Sutton. Learning to predict by the methods of temporal differences. Machine Learning, 3:9-44, 1988.

16. C. J. C. H. Watkins and P. Dayan. Q-learning. Machine Learning, 8:279-292, 1992.

17. M. Wiering and J. Schmidhuber. HQ-learning. Adaptive Behavior, 6(2):219-246, 1998.

18. M.A. Wiering and J. Schmidhuber. Solving POMDPs with Levin search and EIRA. In L. Saitta, editor, Machine Learning: Proceedings of the Thirteenth International Conference, pages 534-542. Morgan Kaufmann Publishers, San Francisco, CA, 1996.

19. S.W. Wilson. ZCS: A zeroth level classifier system. Evolutionary Computation, 2:1-18, 1994. 